\title{
CrystEngComm
}

Check for updates

Cite this: CrystEngComm, 2021, 23, 8215

Received 26th August 2021 ,

Accepted 2nd November 2021

DOI: 10.1039/d1ce01151k

rsc.li/crystengcomm

\section{Tuning the structure of cerium phosphate nanorods $\uparrow$}

\author{
Lisa Allen, iD $\ddagger^{a}$ Josh A. Davies-Jones, (D)*a Philip R. Davies, (iD*a \\ Sarah King ${ }^{\mathrm{a}}$ and Padraic O'Reilly ${ }^{\mathrm{b}}$
}

\begin{abstract}
The one-pot, shape selective synthesis of cerium phosphate nanorods has been explored and developed to give nanoparticles with aspect ratios between 3-24.8. Studies of the surface with photo induced force microscopy show for the first time that despite the hexagonal phase bulk structure, mixed monoclinic and hexagonal domains are present in the surface layer demonstrating the importance of surface sensitive, nanoscale vibrational spectroscopy in nanoparticle science. There is also evidence that carbonate is adsorbed preferentially on the monoclinic domains, though whether the carbonate is the cause of the domains or merely adsorbs more easily on this structure has not been determined. Co, Ni and Mn-doped cerium phosphate rods were prepared successfully with aspect ratios of $>20$. The introduction of dopant ions has a measurable effect upon the magnetic susceptibility but a more significant effect on the bulk crystal phase of the nanoparticles, introducing up to $~ 30 \%$ monoclinic phosphate in the doped rods.
\end{abstract}

\section{Introduction}

Rare-earth orthophosphates $\left(\mathrm{LnPO}_{4}\right)$ are an important family of functional materials with uses in luminescence ${ }^{1-3}$ catalysis, ${ }^{4,5}$ heat-resistance, ${ }^{6}$ cosmetics, ${ }^{7}$ radioactive waste storage $^{8}$ and electrochemistry. ${ }^{9}$ They often have multiple crystal forms, cerium phosphate for example has two well documented crystal structures: rhabdophane (hexagonal) and monazite (monoclinic) with the hexagonal form usually including structural water, $\left(\mathrm{CePO}_{4} \cdot n \mathrm{H}_{2} \mathrm{O}\right)$ and formed at low temperatures while the monoclinic form is anhydrous and formed at high temperatures. The two forms of cerium phosphate show crystal specific properties, Pusztai et al. ${ }^{10}$ for example, demonstrated differences in the surface area and density of surface acidic sites between the hexagonal and monoclinic forms of cerium phosphate nanorods. They also showed a surface conductivity for the monoclinic form three times greater than that of the hexagonal form, accounted for by the observed differences in surface properties. Similarly, Vinothkumar et al., ${ }^{11}$ synthesised samarium doped cerium phosphate nanorods with monoclinic and hexagonal structures and showed a longer luminescent lifetime for the

\footnotetext{
${ }^{a}$ Cardiff Catalysis Institute, School of Chemistry, Cardiff University, Cardiff, CF10 3AT,UK.E-mail:DaviesJA21@cardiff.ac.uk,daviespr@cardiff.ac.uk

${ }^{b}$ Molecular Vista, 6840 Via Del Oro Suite 110, San Jose, CA 95119, USA

$\dagger$ Electronic supplementary information (ESI) available. See DOI: 10.1039/d1ce01151k \$ Current addresses: Department of Chemistry, University College London, 20 Gordon Street, London, WC1H 0AJ, UK and The Research Complex at Harwell, Rutherford Appleton Laboratory, Harwell Campus, Didcot, Oxfordshire, OX11 OFA, UK.
}

monoclinic form which they attributed to quenching by the structural water in the hexagonal structure. They also showed the monoclinic phosphate to have a higher catalytic activity in the presence of peroxidase which they attributed to a greater ability to create hydroxyl radicals.

Since the tuning of the crystal structure of the rare earth phosphates is often a matter of changing simple synthetic parameters such as $\mathrm{pH}$, temperature, or pressure, ${ }^{10-13}$ these materials offer interesting opportunities to optimise their performance for different applications. This is particularly true for heterogeneous catalysis where surface properties are key but it also applies in situations where growth of one phase on another is necessary. The control of morphology is well documented for ceria nanoparticles, for example HaoXin Mai et al. ${ }^{14}$ prepared cerium oxide nanoparticles as cubes, rods, and polyhedra with different proportions of exposed crystal lattices. The oxygen storage and $\mathrm{CO}$ oxidation capabilities of the nanorod and nanocube morphologies, which were assumed to be dominated by [100] and [110] crystal faces, were significantly better than for the polyhedra which were expected to exhibit mainly [111] facets. Si et al. ${ }^{15}$ reported a similar trend for the water gas shift reaction over ceria supported gold catalysts but in both these cases the surface structure is assumed to be a simple termination of the bulk structure because determining the structure of the upper few monolayers of a nanoparticle is very difficult without high resolution TEM and very good samples.

Our interest in the rare earth phosphates is in their potential use in an optical sensing platform which requires a magnetic character and a coating of a plasmonic material. 
The cerium phosphates are ideal for this application because of their well-controlled morphology and weak paramagnetism which can be manipulated in the same way that Xia et $a l .{ }^{16}$ greatly increased the magnetic susceptibility of micron sized cerium oxide nanorods through the addition of $\mathrm{Mn}$ ions into the lattice. In this report, we investigate the morphology of cerium phosphate nanorods under different synthesis conditions and explore the possibility of increasing the magnetic strength of the nanoparticles by doping with other transition metals. We have a particular interest in the surfaces of the rods since the properties of the surface are critical to the effectiveness of the coating procedures we will report in a future publication. In order to study this aspect, we have employed the relatively new photo induced force microscopy (PiFM) technique which provides surface vibrational spectroscopy together with nanometre resolved topography. ${ }^{17,18}$ We show that this allows the mapping of surface structural domains on the cerium phosphate nanorods and importantly a correlation between different domains and adsorbed species under ambient conditions which has great potential for exploring the role surface structure plays in determining surface reaction mechanisms in areas as diverse as coatings, adhesion and catalysis.

\section{Methods}

\subsection{Nanoparticle preparation}

The cerium phosphate nanoparticles were synthesised in a novel procedure designed for use with a sealed glass flask whereas methods published previously have used an autoclave. ${ }^{19} 0.56 \mathrm{~g} \mathrm{Ce}\left(\mathrm{NO}_{3}\right)_{3} \cdot 6 \mathrm{H}_{2} \mathrm{O}$ (Sigma Aldrich, 99.99\%) was added to $40 \mathrm{~mL}$ of $\mathrm{Na}_{3} \mathrm{PO}_{4}$ (Sigma Aldrich, 99.0\%) solution, with a concentration ranging from $8.25 \times 10^{-5} \mathrm{M}$ to $8.25 \times 10^{-2} \mathrm{M}$, and stirred at $300 \mathrm{rpm}$ for 5 minutes at room temperature $(\sim 293 \mathrm{~K})$. The white solution was transferred to a $100 \mathrm{~mL}$ sealed glass pressure flask and heated at temperatures between $100-220{ }^{\circ} \mathrm{C}$ for 16 hours. After cooling, the precipitate was washed in a centrifuge with deionised water and dried at $70{ }^{\circ} \mathrm{C}$ overnight in air.

\subsection{Doping of cerium phosphate}

Doping of cerium phosphate nanorods was carried out at the optimised cerium phosphate nanorod conditions of $180{ }^{\circ} \mathrm{C}$ with $8.25 \times 10^{-2} \mathrm{M} \mathrm{KH}_{2} \mathrm{PO}_{4}$. To this, different amounts of dopant were added.

2.2.1 Manganese. $0.7 \mathrm{~g}$ of $\mathrm{Mn}\left(\mathrm{NO}_{3}\right)_{2} \cdot 4 \mathrm{H}_{2} \mathrm{O}$ (Sigma Aldrich, 99.99\%) was added to $50 \mathrm{~mL}$ of deionised water to form a $13.94 \mathrm{mg} \mathrm{mL}^{-1}$ stock solution. In a sealed pressure flask, 0.56 $\mathrm{g}$ of $\mathrm{Ce}\left(\mathrm{NO}_{3}\right)_{3} \cdot 6 \mathrm{H}_{2} \mathrm{O}$ was dissolved in $10 \mathrm{~mL}$ of deionised water and $5 \mathrm{~mL}$ of $\mathrm{NaH}_{2} \mathrm{PO}_{4}$ stock solution added. To this, between 10 and $1300 \mu \mathrm{L}$ of $\mathrm{Mn}\left(\mathrm{NO}_{3}\right)_{2} \cdot 4 \mathrm{H}_{2} \mathrm{O}$ stock solution was added, depending on doping quantity required, and enough deionised water to bring the final volume of the reaction mixture to $20 \mathrm{~mL}$. After sonication, the solution was heated in an oil bath at $180{ }^{\circ} \mathrm{C}$ for 24 hours. The reaction mixture was separated with centrifugation and washed with deionised water and ethanol then dried at $80^{\circ} \mathrm{C}$ overnight.

2.2.2 Cobalt. $2.1 \mathrm{~g}$ of $\mathrm{Co}\left(\mathrm{NO}_{3}\right)_{2} \cdot 6 \mathrm{H}_{2} \mathrm{O}$ (Sigma Aldrich, $98.0 \%$ ) was added to $50 \mathrm{~mL}$ of deionised water to form a 41.6 $\mathrm{mg} \mathrm{mL}^{-1}$ stock solution. In a sealed pressure flask, $0.56 \mathrm{~g}$ of $\mathrm{Ce}\left(\mathrm{NO}_{3}\right)_{3} \cdot 6 \mathrm{H}_{2} \mathrm{O}$ was dissolved in $10 \mathrm{~mL}$ of deionised water and a $5 \mathrm{~mL}$ aliquot of $\mathrm{NaH}_{2} \mathrm{PO}_{4}$ stock solution was added. To this, between $50-500 \mu \mathrm{L}$ of $\mathrm{Co}\left(\mathrm{NO}_{3}\right)_{2} \cdot 6 \mathrm{H}_{2} \mathrm{O}$ stock solution was added depending on doping level with deionised water to bring the final volume of the reaction mixture to $20 \mathrm{~mL}$. After sonication, the solution was heated in an oil bath at $180{ }^{\circ} \mathrm{C}$ for 24 hours. The reaction mixture was separated with centrifugation and washed with deionised water and ethanol before being dried at $80{ }^{\circ} \mathrm{C}$ overnight.

2.2.3 Nickel. $2.0 \mathrm{~g}$ of $\mathrm{Ni}\left(\mathrm{NO}_{3}\right)_{2} \cdot 6 \mathrm{H}_{2} \mathrm{O}$ (Sigma Aldrich, 98.5\%) was added to $50 \mathrm{~mL}$ of deionised water to form a 40.6 $\mathrm{mg} \mathrm{mL} \mathrm{m}^{-1}$ stock solution. In a sealed pressure flask, $0.56 \mathrm{~g}$ of $\mathrm{Ce}\left(\mathrm{NO}_{3}\right)_{3} \cdot 6 \mathrm{H}_{2} \mathrm{O}$ was dissolved in $10 \mathrm{~mL}$ of deionised water and $5 \mathrm{~mL}$ of $\mathrm{NaH}_{2} \mathrm{PO}_{4}$ stock solution was added. To this, between $45-500 \mu \mathrm{L}$ of $\mathrm{Ni}\left(\mathrm{NO}_{3}\right)_{2} \cdot 6 \mathrm{H}_{2} \mathrm{O}$ stock was added depending on the doping amount, with deionised water added to bring the final volume of the reaction mixture to 20 $\mathrm{mL}$. After sonication for 5 minutes the solution was left to heat in an oil bath at $180{ }^{\circ} \mathrm{C}$ for 24 hours. The reaction mixture was separated with centrifugation and washed with deionised water and ethanol before being dried at $80{ }^{\circ} \mathrm{C}$ overnight.

2.2.4 Characterisation. A Renishaw inVia confocal microscope (Miskin, Wales, UK) was used to record the Raman spectra using a $514 \mathrm{~nm}$ laser and spectra were collected in reflective mode. For XPS the dried nanoparticles were pressed onto conductive tape and analysed with a Kratos Axis Ultra-DLD photoelectron spectrometer with a monochromatic aluminium $\mathrm{K} \alpha \mathrm{X}$-ray source in the "hybrid spectroscopy" mode with an analysis area of $700 \times 300 \mu \mathrm{m}$. A pass-energy of $40 \mathrm{eV}$ was used for high-resolution scans and $60 \mathrm{eV}$ for survey scans. CasaXPS (version 2.3.24) was used to analyse the spectra ${ }^{20}$ with binding energies referenced to the largest C(1 s) peak at $284.7 \mathrm{eV}$ with an uncertainty of $\sim 0.2 \mathrm{eV}$. Raw spectra were modified using Wagner sensitivity factors, as supplied by the instrument manufacturer after the subtraction of a Shirley background. Powder X-ray diffraction (XRD) data over the range $2 \theta=10-80^{\circ}$ were obtained using a PANalytical X'Pert Pro diffractometer with a monochromatic $\mathrm{Cu} \mathrm{K} \alpha$ source $(\lambda=0.154 \mathrm{~nm})$ operated at $40 \mathrm{kV}$ and $40 \mathrm{~mA}$.

Photo induced force microscopy (PiFM) $)^{21,22}$ is a relatively new spectroscopic tool in which dipole-dipole coupling between a surface oscillator and a gold covered AFM tip exerts a measurable force that is detected mechanically. This enables the recovery of near-field vibrational spectra of the surface region that match exactly far-field infrared spectra but are obtained at spacial resolutions well below the diffraction limit. Whilst PiFM can be run in hyper-spectral mode in which every pixel of an AFM image contains a full infrared spectrum, that approach is very time consuming and a more efficient experimental strategy, and the one adopted here, is 
to either select specific points on an image to record a spectrum or to simultaneously map the topography of a surface with the intensity at a single frequency. Samples of the nano-rods were deposited on mica sheets from aqueous suspension and allowed to dry in air. The samples were sent to California for analysis by Molecular Vista where they were examined in air without further sample preparation. The spectroscopic data was analysed using CasaXPS ${ }^{20}$ and fitted using Gaussian curves to clarify the changes that occur between different points in the images, details of the fitting procedure are described below.

\section{Results and discussion}

\subsection{Synthesis: effects of phosphate concentration and synthesis temperature}

The $\mathrm{KH}_{2} \mathrm{PO}_{4}$ or $\mathrm{Na}_{3} \mathrm{PO}_{4}$ concentration has a significant effect on the morphology of the cerium nanoparticles produced by the hydrothermal synthesis, Fig. 1, with a transition from predominantly nanocubes $(\sim 200 \mathrm{~nm}$ wide $)$ to anisotropic nanorods as the phosphate concentration is increased. In the intermediate stages, the TEM images resemble those of $\mathrm{Li}$ et $a{ }^{23}$ but we cannot determine from the TEM whether the association of the nanorods with the cubes is due to aggregation or to the growth of one from the other. As the phosphate concentration is increased, the diameter and length of the nanorods increases from 14.2 to $20.3 \mathrm{~nm}$ and 43.1 to $503.6 \mathrm{~nm}$ respectively, giving an increase in aspect ratio from 3 to 24.8. XP spectra of the Ce3d region (Fig. S1 $\dagger$ ) shows a corresponding transition from $100 \% \mathrm{Ce}(\mathrm{Iv})$ at the lowest phosphate concentrations, through a mixture of states between phosphate concentrations of $3 \times 10^{-4}$ and $5 \times 10^{-2} \mathrm{M}$, to $100 \% \mathrm{Ce}(\mathrm{III})$ at $8.25 \times 10^{-2} \mathrm{M}$ and a Ce $: \mathrm{P}: \mathrm{O}$ ratio of $1: 1: 4$.
The data is consistent with a switch from $\mathrm{CeO}_{2}$ nanocubes to $\mathrm{CePO}_{4}$ nanorods as the phosphate concentration is increased.

The temperature at which the synthesis is carried out, also influences the morphology of the nanoparticles. We found two temperature regimes of interest at a phosphate concentration of $8.25 \times 10^{-2} \mathrm{M}$. For $\leq 180{ }^{\circ} \mathrm{C}$ we produced exclusively cerium phosphate nanorods, where increasing the temperature in increments up to $180{ }^{\circ} \mathrm{C}$ led to a growth in the rod length/aspect ratio. At synthesis temperatures $>200$ ${ }^{\circ} \mathrm{C}$, the production of large $(\geq 600 \mathrm{~nm})$ polyhedra dominates. In the lower temperature regime, TEM shows that the rods grow preferential along the [001] axis with a hexagonal crystal structure. The rod length grows with increasing temperature, Fig. 2, from an average length of 98.7 to $795.6 \mathrm{~nm}$ with an apparent activation energy of $26.5 \mathrm{~kJ} \mathrm{~mol}^{-1}$, Fig. S2, $\dagger$ suggesting a process limited by diffusion of cerium intermediates to the ends of the growing rods.

The XRD data in Fig. 3, confirm the transition from cerium oxide to cerium phosphate as the phosphate concentration is increased, with the particles formed at lower potassium phosphate concentrations displaying XRD patterns corresponding only to that of cerium oxide with intense [111] and [220] peaks characteristic of a cubic fluorite $F \bar{m} 3 m$ space group. At higher phosphate concentrations, 12 peaks are observed corresponding to the $\mathrm{P6}_{2} 22$ space group of hexagonal $\mathrm{CePO}_{4}$ with the [200], [102] and [211] peaks being the most intense.

Raman spectra of the rods, Fig. 4, show a typical $\mathrm{CeO}_{2}$ pattern at a phosphate concentration of $0.33 \times 10^{-3} \mathrm{M}$ with an intense $\mathrm{F}_{2 \mathrm{~g}}$ first order phonon at $459 \mathrm{~cm}^{-1}$ corresponding to the fluorite crystal system, and two weak peaks at 260 $\mathrm{cm}^{-1}$ and $1065 \mathrm{~cm}^{-1}$ caused by the second-order transverse acoustic mode (2TA) and a second-order longitudinal optical mode (2LO). ${ }^{24}$ A weak $\mathrm{PO}_{4}{ }^{-}$symmetric stretching band at 979

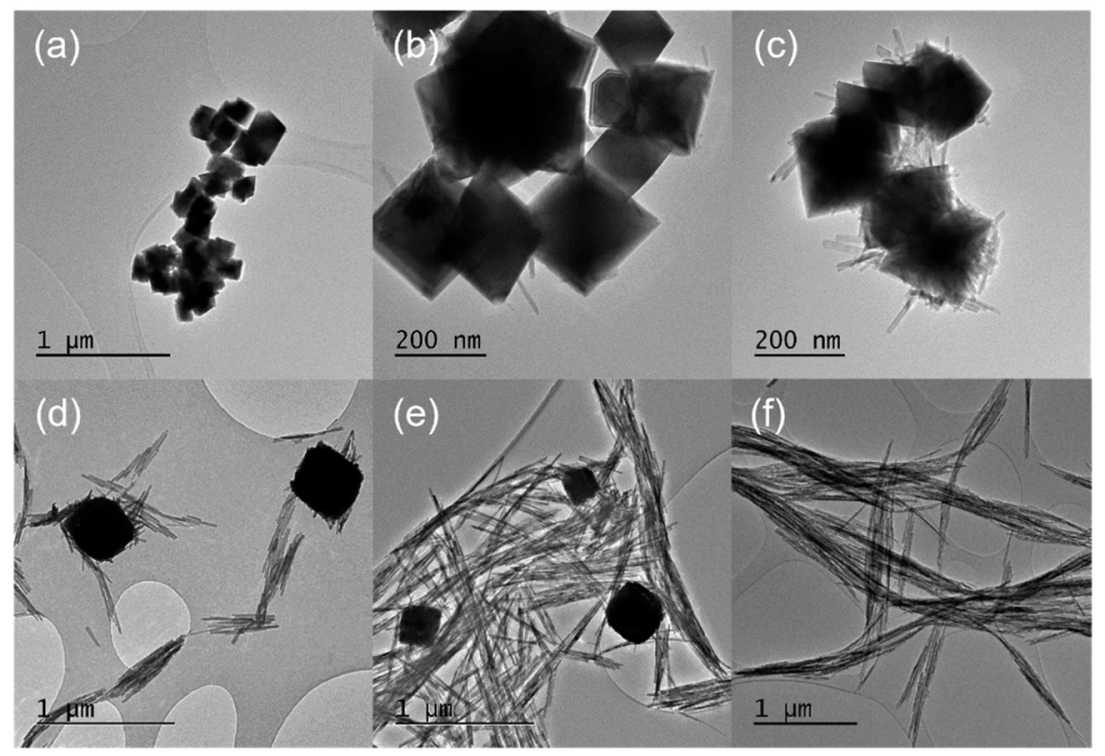

Fig. 1 TEM micrographs of cerium nanostructures formed with varying concentrations of phosphate at $170{ }^{\circ} \mathrm{C}$ (a) $8.25 \times 10^{-5} \mathrm{M}$; (b) $3.3 \times 10^{-4} \mathrm{M}$; (c) $4.4 \times 10^{-4} \mathrm{M}$; (d) $1.65 \times 10^{-3} \mathrm{M}$; (e) $4.125 \times 10^{-2} \mathrm{M}$; (f) $8.25 \times 10^{-2} \mathrm{M}$. 


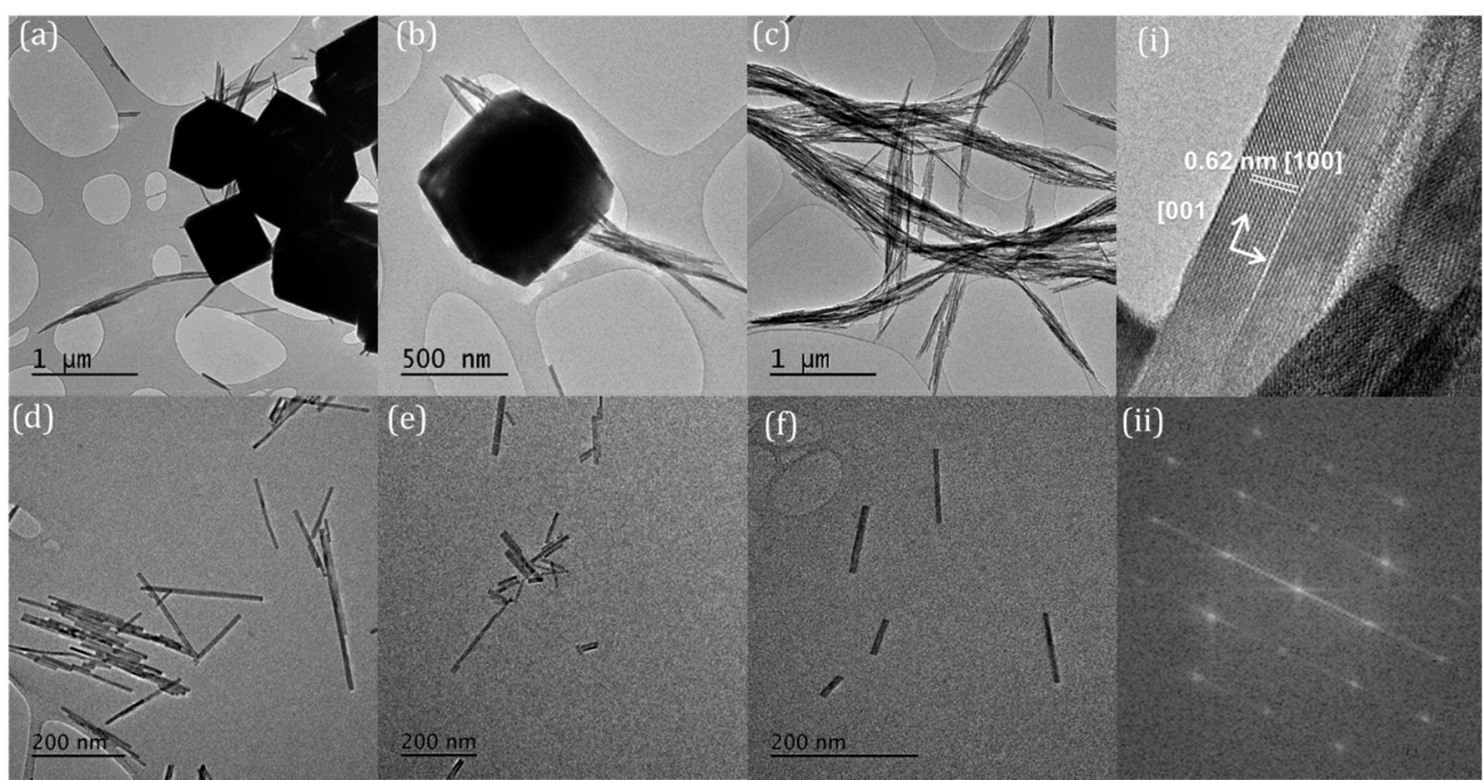

Fig. 2 TEM micrographs of cerium nanoparticles formed at (a) $220^{\circ} \mathrm{C}$; (b) $200{ }^{\circ} \mathrm{C}$; (c) $180{ }^{\circ} \mathrm{C}$; (d) $140{ }^{\circ} \mathrm{C}$; (e) $120{ }^{\circ} \mathrm{C}$; (f) $100{ }^{\circ} \mathrm{C}$; (i) $170{ }^{\circ} \mathrm{C}$ with axis labelled; (ii) the FFT of (i) showing preferential growth along the [001] axis and a hexagonal crystal structure.
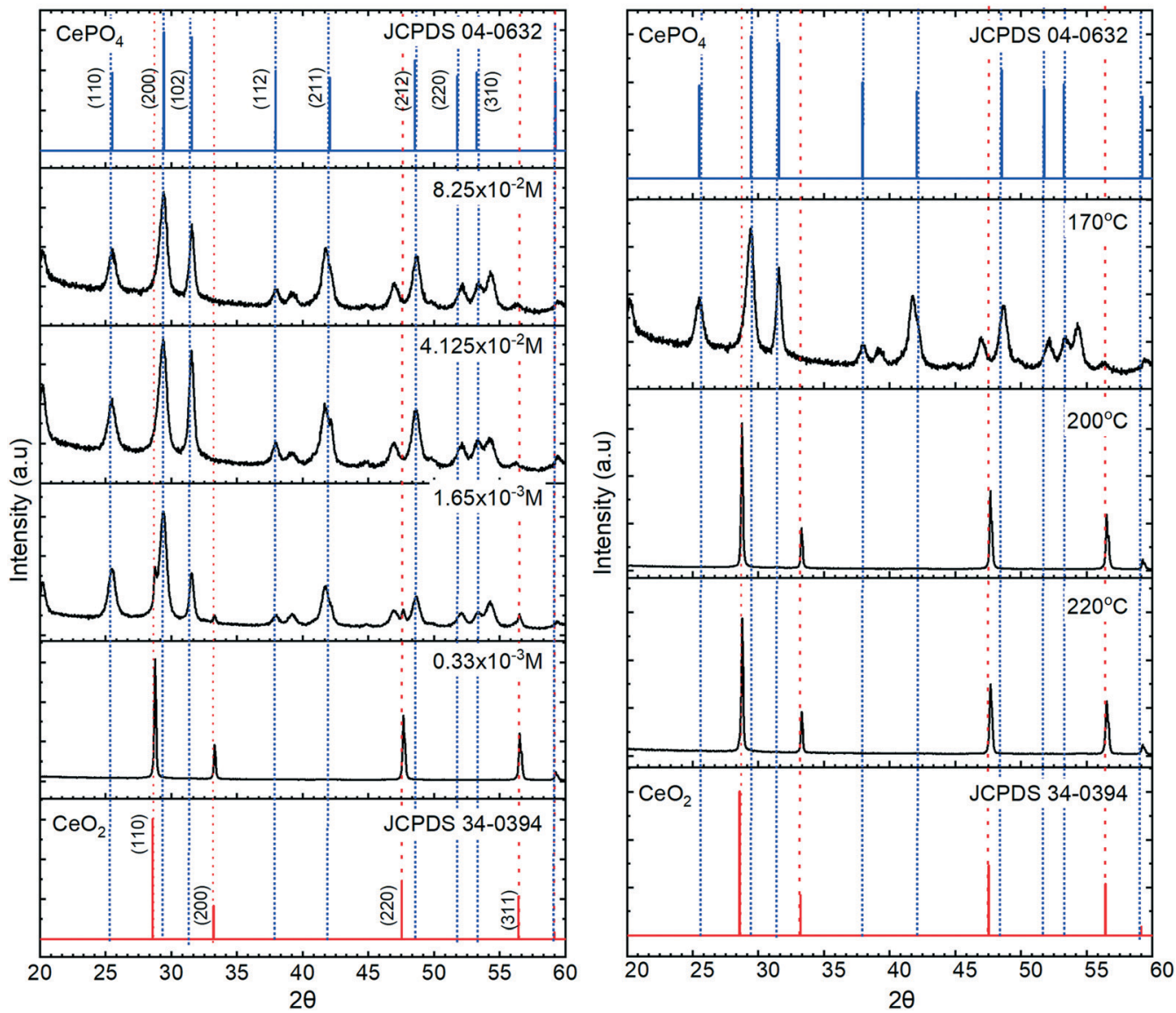

Fig. 3 Left, XRD patterns of cerium phosphate nanoparticles obtained at different concentrations of phosphate with reference data from the ICDMS data base for $\mathrm{CeO}_{2}$ and hexagonal $\mathrm{CePO}_{4}$ shown in blue and red, respectively on both charts. Right, XRD for cerium nanoparticles prepared at $220,200,170{ }^{\circ} \mathrm{C}$ with $8.25 \times 10^{-2} \mathrm{M}$ potassium phosphate. 


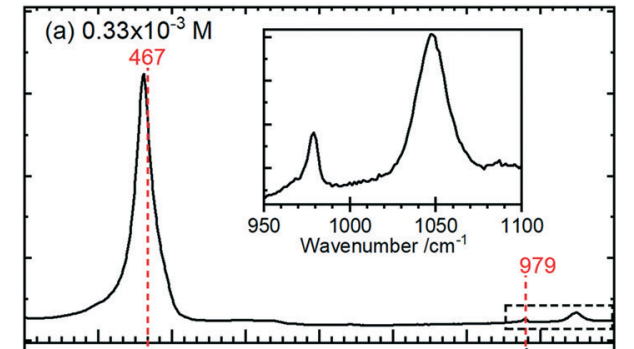

(b) $4.125 \times 10^{-2} \mathrm{M}$

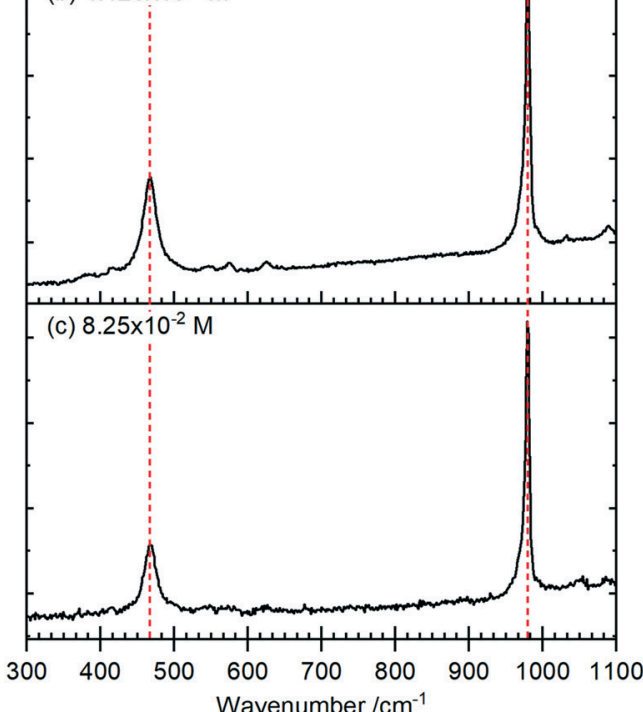

Fig. 4 Raman spectra for cerium nanoparticles formed with different concentrations of potassium phosphate (a) $0.33 \times 10^{-3} \mathrm{M}$; (b) $4.125 \times$ $10^{-2} \mathrm{M}$; (c) $8.25 \times 10^{-2} \mathrm{M}$. The inset in (a) is a magnification of the $\mathrm{PO}_{4}$ stretching region indicated by the dashed rectangle.

$\mathrm{cm}^{-1}$ can also be detected, indicating the presence of some hexagonal structured cerium phosphate in the sample,

At higher concentrations of phosphate the Raman signal due to the ceria all but disappears (most clearly indicated by the loss of intensity in the $1050 \mathrm{~cm}^{-1}$ peak in (a)) and is replaced by Raman bands at $979 \mathrm{~cm}^{-1}$ and $467 \mathrm{~cm}^{-1}$ that can be assigned to the $\mathrm{V}_{1}$ and $\mathrm{V}_{2}, \mathrm{PO}_{4}$ stretching and bending modes of hexagonal $\mathrm{CePO}_{4}$ along with weaker peaks at $1085 \mathrm{~cm}^{-1}, 627 \mathrm{~cm}^{-1}$ and 573
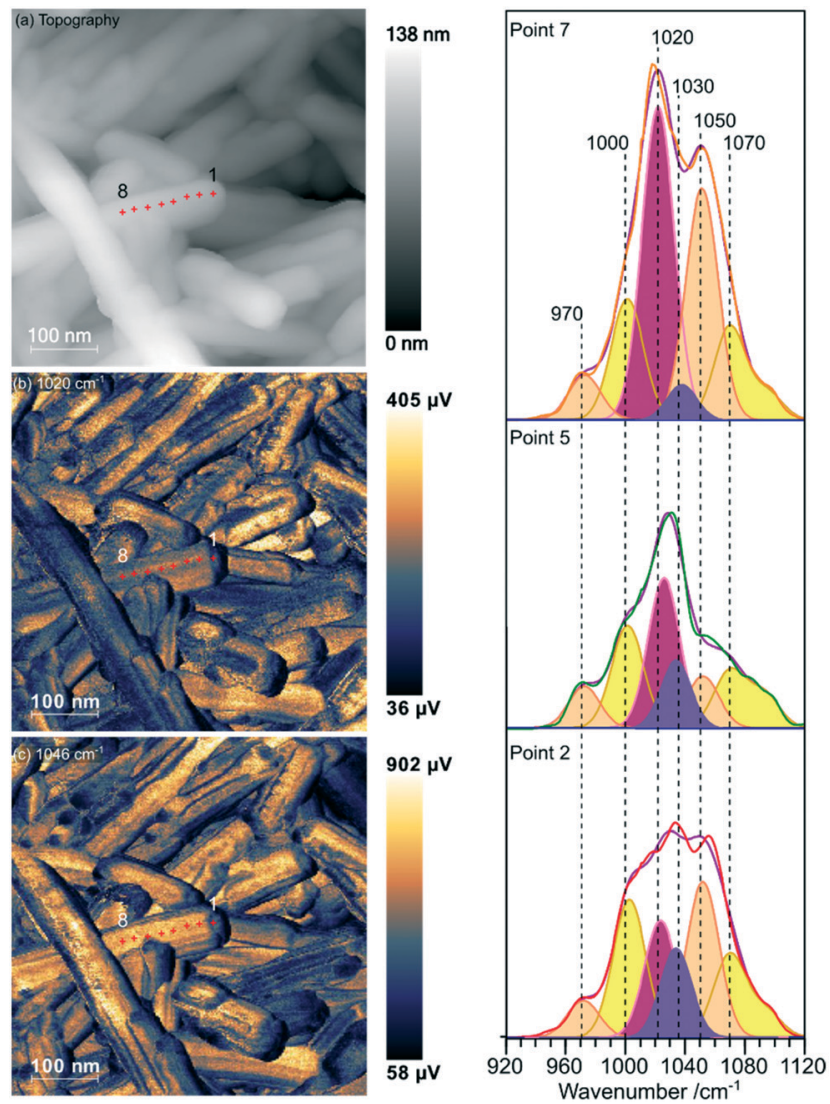

Fig. 5 PiFM images and spectra of cerium phosphate nanorods synthesised at a phosphate concentration of $8.25 \times 10^{-2} \mathrm{M}$ and a temperature of $170^{\circ} \mathrm{C}$. (a) Topography; (b) and (c) maps of the vibrational intensity measured by PiFM at 1020 and $1046 \mathrm{~cm}^{-1}$ respectively selected to contrast the presence of hexagonal structure, see text. (Right) PiFM spectra obtained from positions 2, 5 and 7 marked on the topography and PiFM maps. The $V_{1}$ and $V_{3}$ region is shown with the curve fitted peaks coloured according to the species to which they are assigned (light brown = hexagonal $\mathrm{CePO}_{4}$, yellow $=$ monoclinic $\mathrm{CePO}_{4}$, pink = monoclinic and hexagonal, purple $=$ carbonate). Note: in the figure, adjacent monoclinic components are combined for clarity. Full PiFM spectra are shown in Fig. S4.†

$\mathrm{cm}^{-1}$, Table 1. Finally, there is evidence for the presence of some monoclinic phase in the samples with weak features at $414 \mathrm{~cm}^{-1}$,

Table 1 Experimentally observed IR and Raman values for monoclinic and hexagonal cerium phosphates ${ }^{25}$

\begin{tabular}{|c|c|c|c|c|c|}
\hline \multicolumn{3}{|c|}{ IR-spectroscopy wavenumber $/ \mathrm{cm}^{-1}$} & \multicolumn{3}{|c|}{$\underline{\text { Raman - spectroscopy wavenumber } / \mathrm{cm}^{-1}}$} \\
\hline & 1104 & $V_{3}$ & 1085 & 1070 & $\mathrm{~V}_{3}$ \\
\hline 1052 & 1062 & & & 1024 & \\
\hline \multirow{2}{*}{1020} & 1025 & & & 990 & \\
\hline & 995 & & & & \\
\hline & & & 624 & 618 & $\mathrm{~V}_{4}$ \\
\hline 615 & 623 & $\mathrm{~V}_{4}$ & 573 & & \\
\hline 569 & 579 & & & & \\
\hline \multirow[t]{2}{*}{542} & 567 & & 466 & 466 & $\mathrm{~V}_{2}$ \\
\hline & 542 & & 377 & 414 & \\
\hline
\end{tabular}



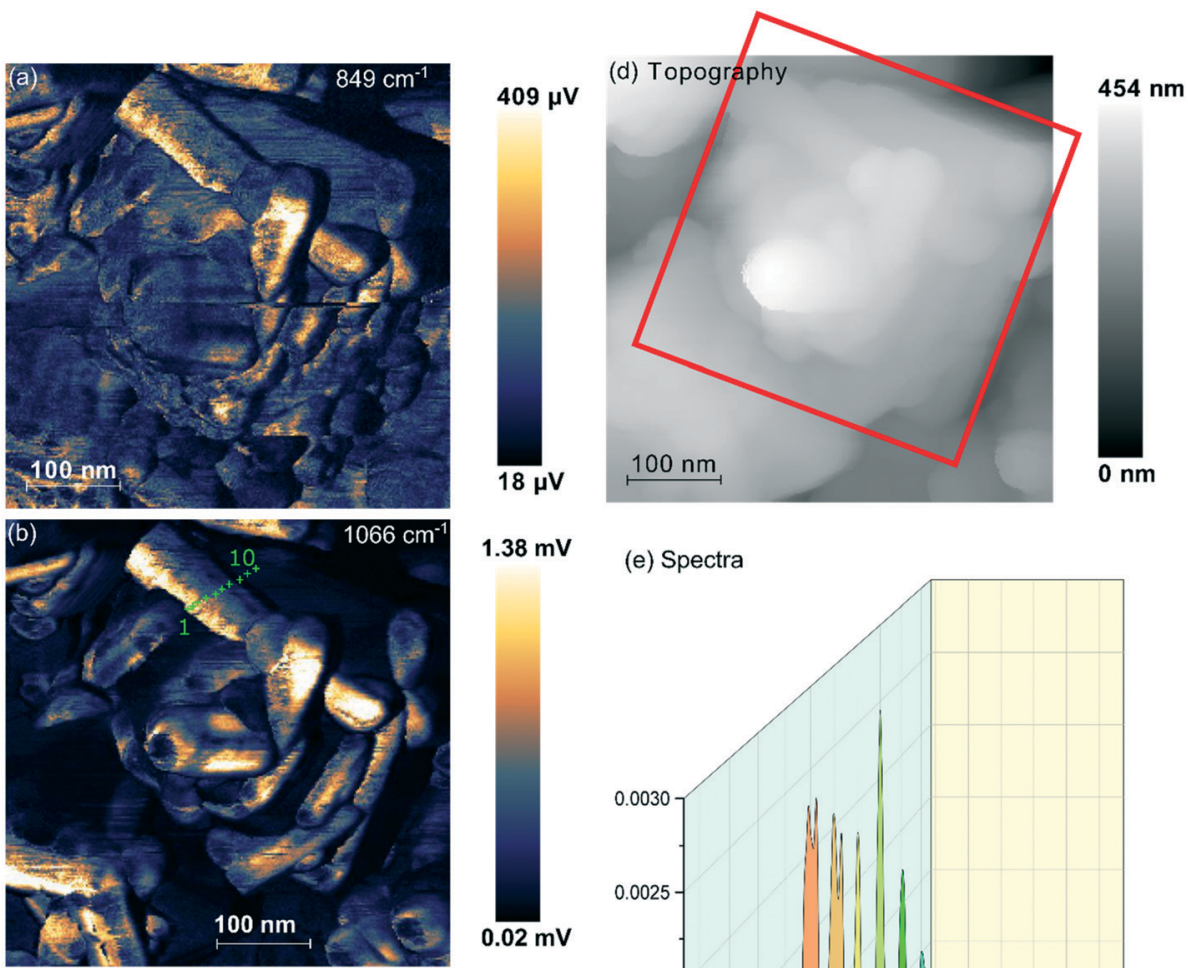

$1.38 \mathrm{mV}$

(e) Spectra
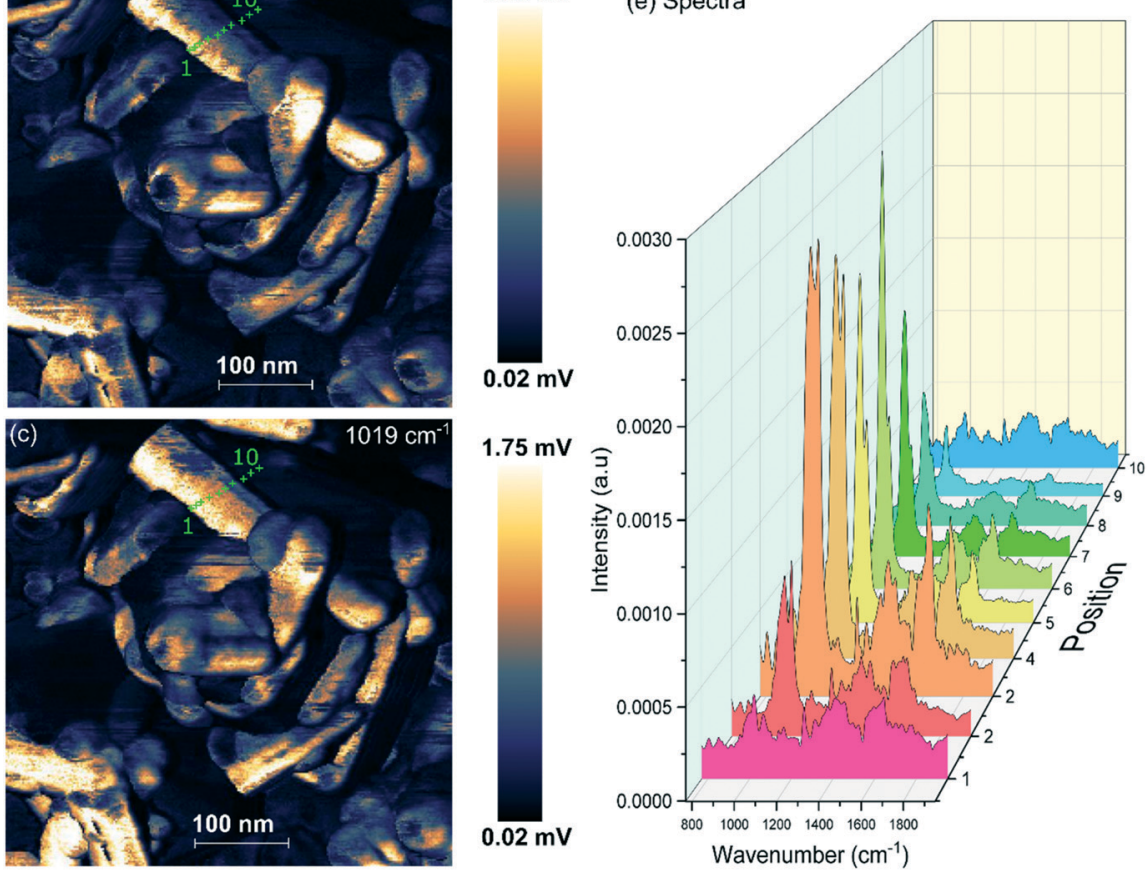

Fig. 6 PiFM images and spectra of cerium phosphate nanorods synthesised at a phosphate concentration of $4.4 \times 10^{-3} \mathrm{M}$ and a temperature of $170{ }^{\circ} \mathrm{C}$. PiFM intensity maps at: (a) $849 \mathrm{~cm}^{-1}$; (b) $1066 \mathrm{~cm}^{-1}$; and (c) $1019 \mathrm{~cm}^{-1}$; (d) topography, red square indicates the outline of a CeO $\mathrm{Cube}$; (e) waterfall plot of PiFM spectra across the surface (points 1-10).

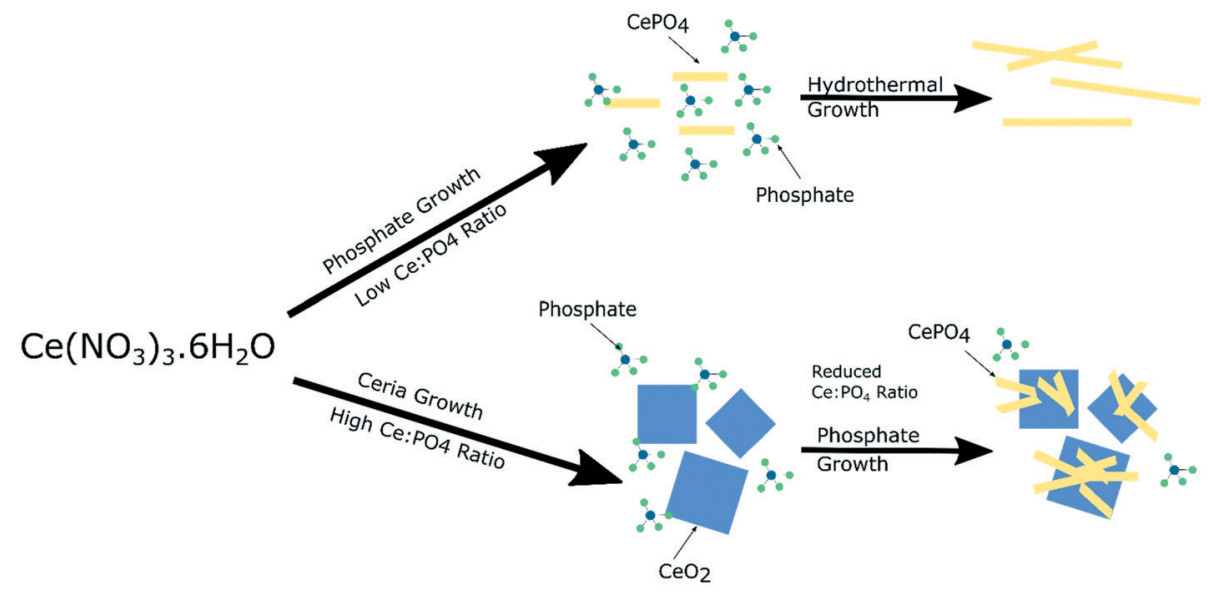

Fig. 7 Illustration of suggested growth mechanism of cerium nanoparticles based on phosphate concentration. 


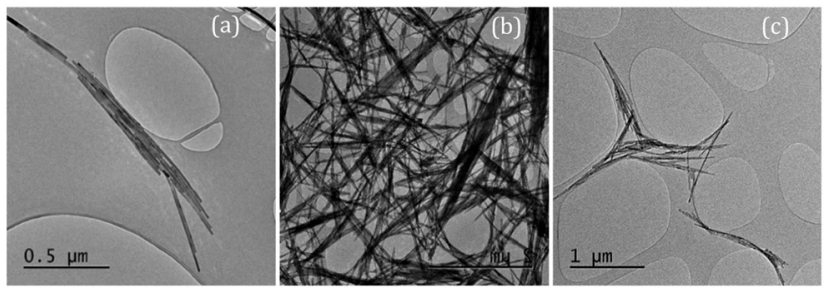

Fig. 8 TEM images of cerium phosphate nanorods synthesised at 180 ${ }^{\circ} \mathrm{C}$ and doped with $0.15 \mathrm{wt} \%$ (a) manganese; (b) cobalt, and (c) nickel.

$1050 \mathrm{~cm}^{-1}$ and a pronounced asymmetry to the low wavenumber side of the $979 \mathrm{~cm}^{-1}$ peak. The features assigned to monoclinic phosphate are more apparent in the Raman spectra of samples prepared at higher temperatures (Fig. S3†).

Samples of undoped rods formed at higher concentrations of phosphate, and those with mixed morphology were examined with the local spectroscopic tool PiFM. Spectra were obtained at specific points on the nanoparticles between 770 and 1950 $\mathrm{cm}^{-1}$, and intensity maps at selected wavenumbers were also collected, providing a unique insight into the composition of the surface region of the particles. In the discussion below we concentrate mainly on the $900-1200 \mathrm{~cm}^{-1}$ region of the spectrum which can contain components from both monoclinic and hexagonal cerium phosphate. To identify the components involved in the complex envelopes the data was fitted with Gaussian peak shapes with FWHM of $20-25 \mathrm{~cm}^{-1}$. A minimum of 7 peaks is required to give good fits in the $900-1200 \mathrm{~cm}^{-1}$ region of all the spectra. The fitting procedure allowed a variation of $\sim \pm 5 \mathrm{~cm}^{-1}$ in position for each peak around the position of the main components expected for the infrared spectra of hexagonal and monoclinic structures. Good fits were obtained with peaks at 971.9 and $1050 \mathrm{~cm}^{-1}$ corresponding to hexagonal vibrations (coloured light brown in Fig. 5) and 1001.8, 1072.6 and $1093.7 \mathrm{~cm}^{-1}$ corresponding to monoclinic vibrations, (coloured yellow in Fig. 5). A sixth component at $\sim 1020 \mathrm{~cm}^{-1}$ (coloured pink) occurs in both structures and the final component at $1036.0 \mathrm{~cm}^{-1}$ (purple) doesn't match well with any of the expected phosphate vibrations, Table 1. However, the 1036 peak correlates well with other peaks observed at 1410, 1390-1365, and $850 \mathrm{~cm}^{-1}$ which match those expected for a bidentate carbonate at cerium surfaces. ${ }^{26}$

Maps of intensity at 1020 and $1046 \mathrm{~cm}^{-1}$ show a consistent state along the rod and the fitting of the spectra reflect this with only small changes in the relative amounts of hexagonal and monoclinic phases between points.

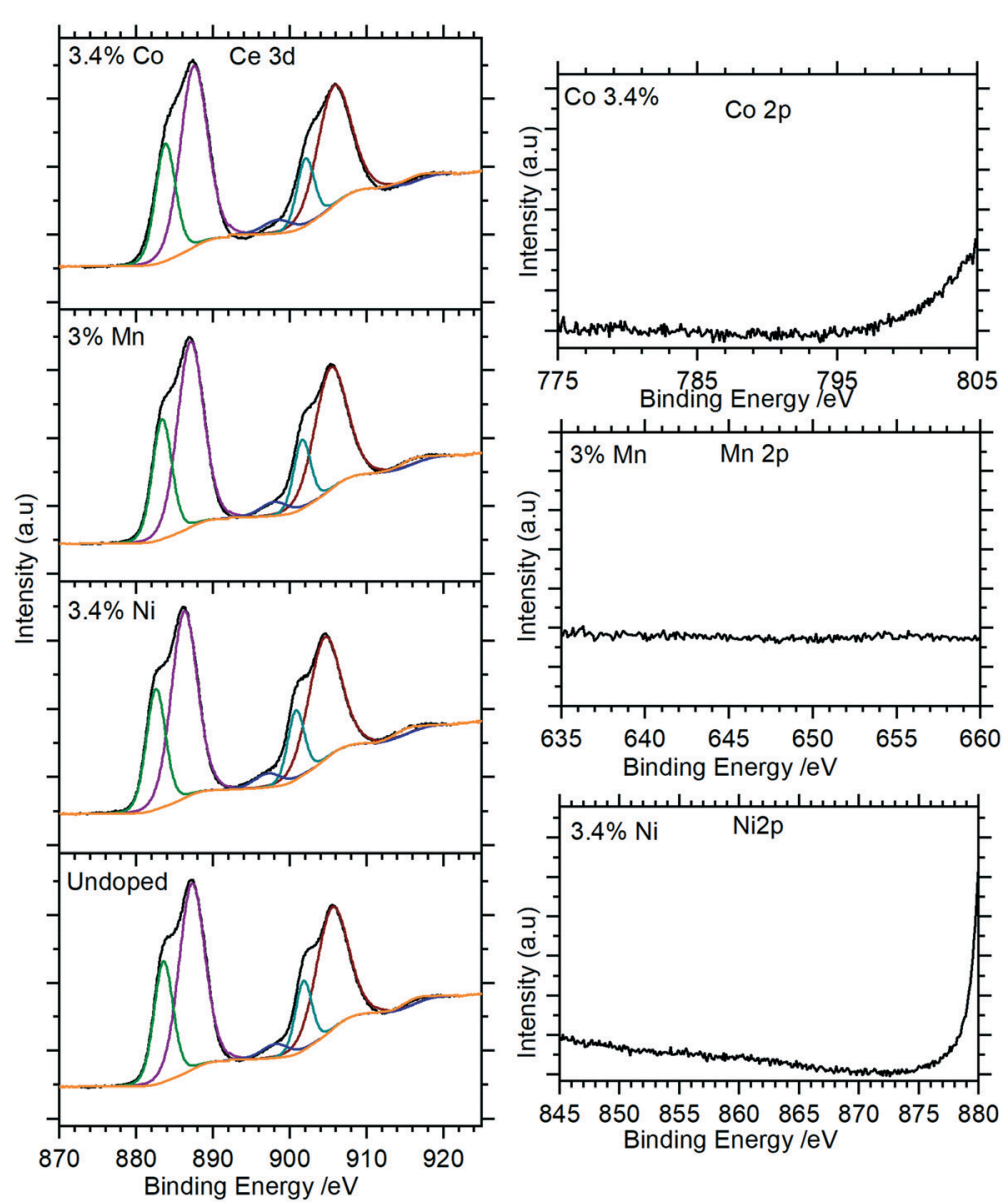

Fig. 9 XP spectra of Co doped cerium phosphate nanoparticles. Left: Ce(3d) spectra for selected samples; right: corresponding region for dopant. Complete spectra for all samples are shown in Fig. S5.† 

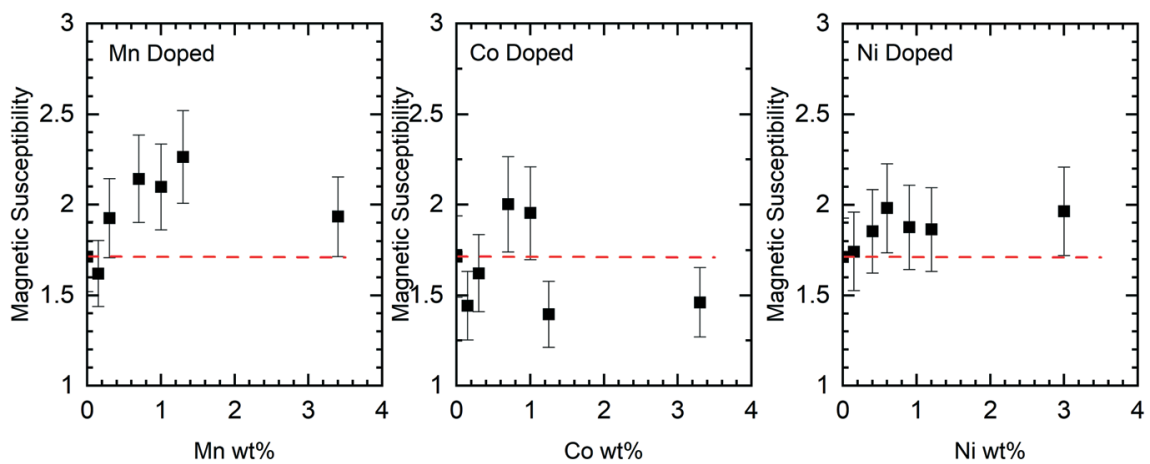

Fig. 10 Graphs of the average molar susceptibility of doped cerium phosphate nanorods as a function of dopant concentration. The dashed line at $1.714 \times 10^{-4}$ $\mathrm{cm}^{3} \mathrm{~g}^{-1}$ represents the average molar susceptibility of the undoped nanorods. Errors were calculated from an average of 3 measurements for each substituent.

The presence of domains of different structure at the surface of the nano rods is nicely demonstrated in Fig. 6 where the vibrational spectrum of the sample is mapped across a rod. The intensity map at $1066 \mathrm{~cm}^{-1}$, Fig. 6(b), shows areas with higher concentrations of monoclinic phosphate whilst the map at $1020 \mathrm{~cm}^{-1}$ corresponds to the peak that is shared by both monoclinic and hexagonal structures, the differences between the two shows areas populated with higher concentrations of the hexagonal structure. From the $1066 \mathrm{~cm}^{-1}$ map its evident that the monoclinic structure is concentrated on the edge of the rod, whereas the map corresponding to the combined hexagonal and monoclinic signal shows intensity right across the rod. The same pattern is evident on several of the other nanorods. Interestingly, the map at $849 \mathrm{~cm}^{-1}$ correlates very well with that of the monoclinic peak. Adsorbed phosphate can show a peak at this position but would be expected to be present at both hexagonal and monoclinic surfaces. The alternative assignment of the $849 \mathrm{~cm}^{-1}$ peak to carbonate was discussed above and is stronger since it correlates well with the other carbonate peaks. That suggests that the carbonate adsorbs preferentially on the monoclinic phosphate.

In addition to the vibrational spectra, the PiFM maps in Fig. 6 also show the relationship between the nanorods of phosphate and the nanocubes of ceria much more clearly than the TEM images in Fig. 1. It's clear from these images that the phosphate rods are embedded in the surface of a ceria cube confirming previous suggestions that the phosphate rods nucleate on the cubes.

\subsection{Growth mechanism}

The local vibrational spectroscopy of PiFM provides some evidence towards the growth mechanism of the cubes and rods. Previous work has shown the formation of cerium(III) hydroxide particles from aqueous solutions of the cerium nitrate, which subsequently undergo a dissolution-recrystallisation process ${ }^{27-29}$ to form the ceria nanoparticles. The evidence here for the growth of the cerium phosphates from the surface of the ceria is consistent with a mechanism in which high concentrations of phosphate ions direct the hydroxide towards the phosphate. At low phosphate concentrations ceria is formed preferentially but as the local cerium concentration is depleted by precipitation, nucleation of $\mathrm{CePO}_{4}$ on the surface of the cerium oxide nanocubes can take place (Fig. 7).

\subsection{Effect of doping on the morphology and magnetism of the $\mathrm{CePO}_{4}$ nanorods}

Cerium phosphate nanorods prepared in the presence of between 0.1 and $3.5 \mathrm{wt} \% \mathrm{Co}^{2+}, \mathrm{Ni}^{2+}$ and $\mathrm{Mn}^{2+}$ ions showed no difference in rod diameter with a consistent average of $19.6 \mathrm{~nm}$ for all the samples. However, the average nanoparticle length was decreased from $430 \mathrm{~nm}$ to 346,312 and $304 \mathrm{~nm}$ for $\sim 3 \mathrm{wt} \% \mathrm{Ni}, \mathrm{Mn}$ and Co respectively (Fig. 8).

XP spectra of the doped nanorods were recorded to investigate the extent to which the dopants were successfully incorporated into the nanorods. As Fig. 9 (and Fig. S5 $†$ ) show, these closely match the spectra of the undoped samples with the $\mathrm{Ce}(3 \mathrm{~d})$ region containing only the features typical of the $\mathrm{Ce}(\mathrm{III})$ in $\mathrm{CePO}_{4}$. There is no evidence for $\mathrm{Ni}, \mathrm{Mn}$, or Co in the XP spectra indicating that the concentrations of these dopants in the surface region are below the detectable limits for XPS ( 1\%) even for those samples with nominal doping levels that are much higher.

Although the XPS data show no evidence for the dopants in the nanorods there is support for them having effects on the rods through other characterisation methods. Changes to the magnetic susceptibility of the nanorods on doping were measurable with a consistent increase for the Mn doped samples but changes that are only just outside the measurement error for the Co and Ni doped samples, Fig. 10.

More concrete evidence for the impact of the dopants is the change in XRD pattern. In the absence of dopants, XRD patterns show a single hexagonal $\mathrm{CePO}_{4}$ phase but inclusion of $\mathrm{Ni}$, Co and $\mathrm{Mn}$ all lead to the development of mixed monoclinic and hexagonal phase systems, (Fig. 11 and S6 $†$ ). Rietveld analysis shows the proportion of the monoclinic phase increases initially up to a maximum of about $40 \%$ but decreases again as the dopant concentration increases (Fig. S7, Table S1†). 

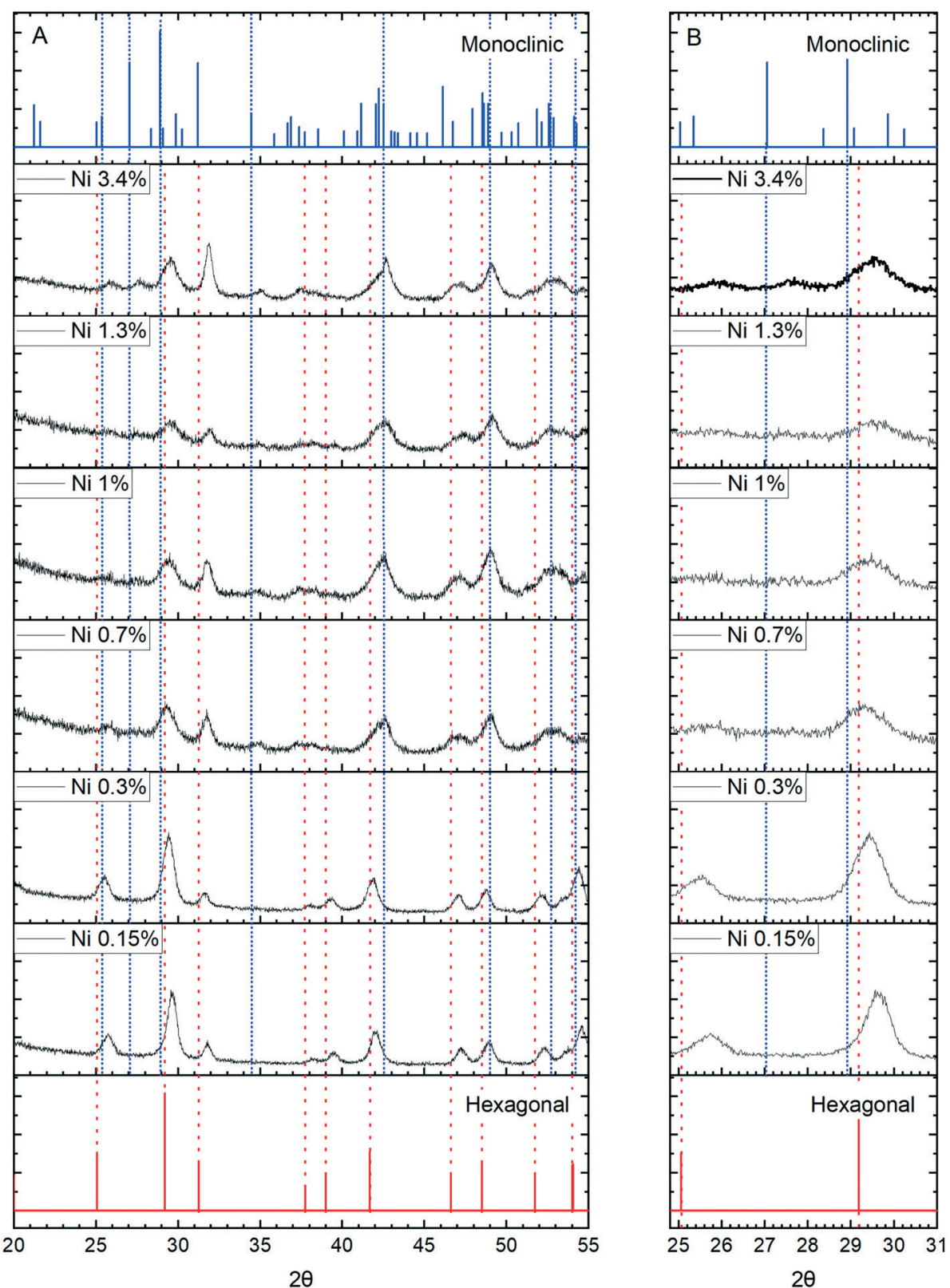

Fig. $11 \mathrm{XRD}$ of nickel doped-cerium nanoparticles prepared with $8.25 \times 10^{-2} \mathrm{M}$ potassium phosphate at $170{ }^{\circ} \mathrm{C}$. The shifts in the precise position of the [200] peak is highlighted in (B) which shows an expansion of (A) along the $2 \theta$ scale, providing evidence for the incorporation of nickel into the cerium phosphate lattice followed by a switch to the monoclinic phase. Reference data from the ICDMS data base for monoclinic (JSPDS 39-0199) and hexagonal $\mathrm{CePO}_{4}$ (ICDD 04-0632) are shown at the top and bottom of the chart respectively.

The appearance of the monoclinic phase could be due to an effect of the ions on the crystallisation process but strong evidence for the incorporation of ions within the lattice comes from shifts of the [200] peak to higher angles for all three dopants, Fig. 11 and S8.†

Raman spectra of the doped nanorod systems show no additional peaks that might be attributed to $\mathrm{Co}, \mathrm{Mn}$ or $\mathrm{Ni}$ components, in agreement with the XPS and XRD, but Fig. 9, does show increased complexity in the $\mathrm{V}_{3}$ peaks between $990-1080 \mathrm{~cm}^{-1}$ indicating the presence of other phases. Deconvolution of the spectra identifies new peaks at 971 and $990 \mathrm{~cm}^{-1}$ which, as discussed in connection to the PiFM data are indicative of the presence of a monoclinic phase supporting the evidence from XRD for the effect of substitution of the dopant ions into the lattice (Fig. 12).

\section{Conclusions}

An investigation of the one pot hydrothermal synthesis of cerium phosphate nanorods using sodium phosphate and cerium nitrate has demonstrated a degree of control over the morphology and chemical composition of the nanorods and an insight into the factors affecting crystal growth in this system. $\mathrm{CePO}_{4}$ nanorods with a $\mathrm{PG}_{2} 22$ space group hexagonal bulk 

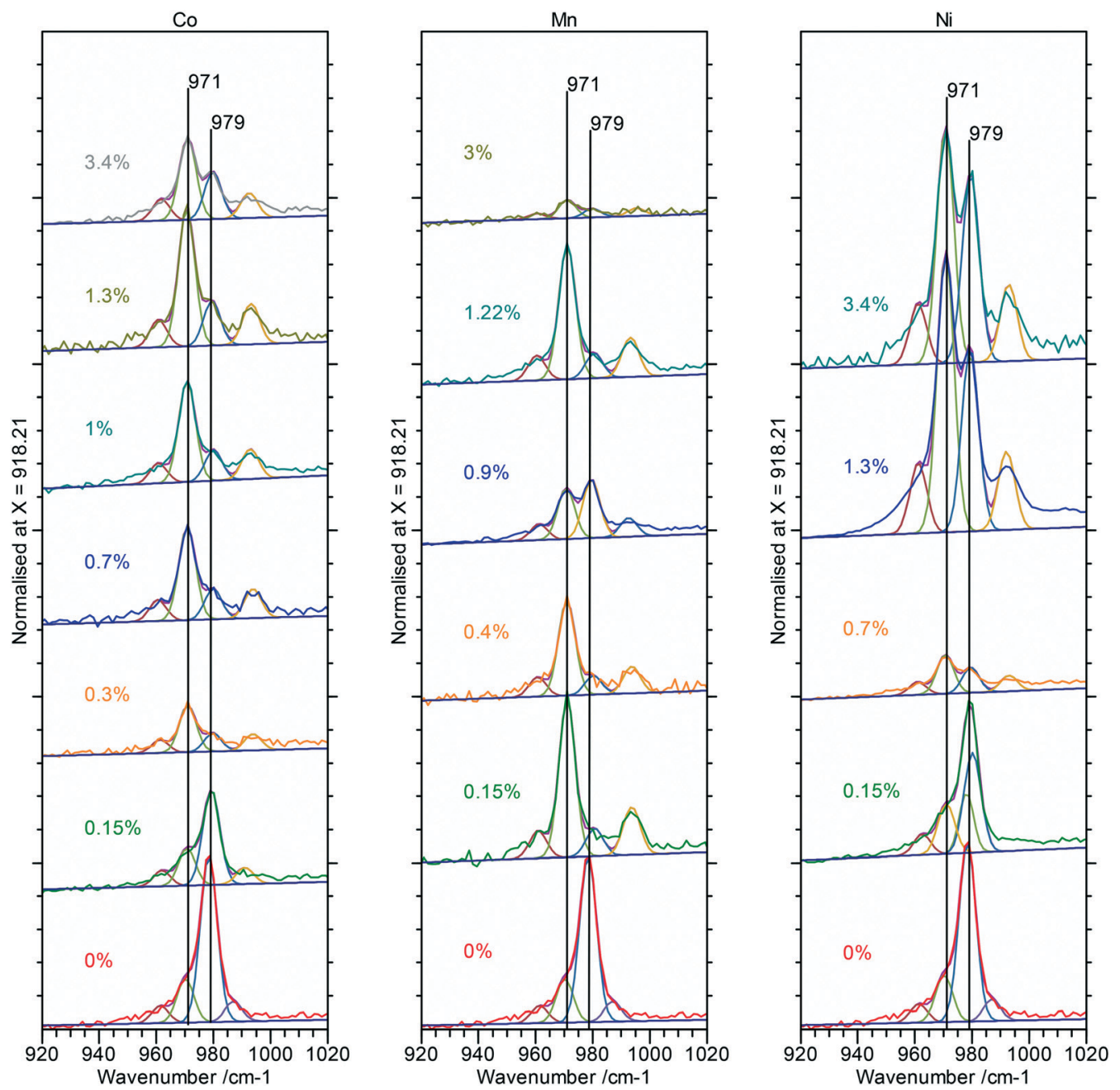

Fig. $12 \mathrm{~V}_{3}$ region of the Raman spectra for $\mathrm{Co}, \mathrm{Mn}$ and Ni doped cerium phosphate rods. Fitting using Gaussian peaks is to guide the eye. The spectra clearly show the enhancement of the monoclinic contribution at $971 \mathrm{~cm}^{-1}$ with dopant concentration.

structure, are formed at temperatures $<200{ }^{\circ} \mathrm{C}$, while ceria nanocubes and polyhedra form at high temperatures and with low concentrations of phosphate. Under conditions where a mixture of ceria and cerium phosphate is produced the PiFM images suggest that the latter nucleates on the ceria cubes.

The method was optimised to give a tuneable aspect ratio for the rods of between 3-26.5. Despite the hexagonal bulk structure, the PiFM spectra show that domains of monoclinic cerium phosphate coexist with the hexagonal structure at the surface of the rods. The spectra also give good evidence for the presence of a surface carbonate associated with the monoclinic domains. This demonstration of the powerful analytical capabilities of surface sensitive vibrational spectroscopy is an important outcome of the investigation. It's not clear from the data whether the surface carbonate is the cause of, or result of, the presence of the monoclinic domains, but this new information is important for work involving the coating of the rods where changes of phase can have a significant impact on the coating growth. The subtle balance between the two phosphate structures is also highlighted by the effect of doping with $\mathrm{Co}^{2+}, \mathrm{Mn}^{2+}$ and $\mathrm{Ni}^{2+}$. All three ions were successfully incorporated into the nanorods (as demonstrated by a change in the lattice spacing of the rods) and whilst they did not have a significant impact on the magnetic properties, they did result in the development of bulk domains of the monoclinic phosphate structure.

\section{Author contributions}

Josh A. Davies-Jones \& Philip R. Davies: conceptualization, data curation, formal analysis, investigation, writing original draft, review \& editing. Lisa Allen, Sarah King and Padraic O'Reilly: investigation, writing - review \& editing.

\section{Conflicts of interest}

There are no conflicts to declare.

\section{Acknowledgements}

JADJ's PhD was funded by Cotton Mouton Diagnostics Ltd and the Knowledge Economy Skills Scholarships (KESS) a pan-Wales higher level skills initiative led by Bangor University on behalf 
of the HE sector in Wales. KESS is part funded by the Welsh Government's European Social Fund (ESF) convergence programme for West Wales and the Valleys. XP spectra were acquired by EPSRC National Facility for Photoelectron Spectroscopy (HarwellXPS), operated by Cardiff University and UCL under contract number PR16195. The authors are grateful to Molecular Vista for access to the PiFM system and to Ian Holton of Acutance Scientific Ltd for his help with the PiFM and many stimulating discussions.

\section{References}

1 P. Xu, R. Yu, L. Zong, J. Wang, D. Wang, J. Deng, J. Chen and X. Xing, J. Nanopart. Res., 2013, 15, 1622.

2 S. Lin, Y. Yuan, H. Wang, R. Jia, X. Yang and S. Liu, J. Mater. Sci.: Mater. Electron., 2009, 20, 899-904.

3 W. Di, X. Zhao, Z. Nie, X. Wang, S. Lu, H. Zhao and X. Ren, J. Lumin., 2010, 130, 728-732.

4 S. Navarro-Jaén, M. Á. Centeno, O. H. Laguna and J. A. Odriozola, J. Mater. Chem. A, 2018, 6, 17001-17010.

5 A. Kumar and R. Srivastava, Sustainable Energy Fuels, 2019, 3, 2475-2489.

6 C. Thiriet, R. J. M. Konings, P. Javorský and F. Wastin, Phys. Chem. Miner., 2004, 31, 347-352.

7 H. Onoda and R. Tanaka, J. Mater. Res. Technol., 2019, 8, 5524-5528.

8 K. Jolley, R. Asuvathraman and R. Smith, Nucl. Instrum. Methods Phys. Res., Sect. B, 2017, 393, 93-96.

9 J. Wang, X. Wu, X. Cao, Y. Jiang, D. Zhang, T. Yang, F. Zhang and Y. Luo, CrystEngComm, 2020, 22, 1610-1618.

10 P. Pusztai, H. Haspel, I. Y. Tóth, E. Tombácz, K. László, Á. Kukovecz and Z. Kónya, ACS Appl. Mater. Interfaces, 2015, 7, 9947-9956.

11 G. Vinothkumar, I. L. Arun, P. Arunkumar, W. Ahmed, S. Ryu, S. W. Cha and K. S. Babu, J. Mater. Chem. B, 2018, 6, 6559-6571.

12 Y. P. Fang, A. W. Xu, A. M. Qin and R. J. Yu, Cryst. Growth Des., 2005, 5, 1221-1225.

13 A. Ochiai and S. Utsunomiya, Minerals, 2017, 7, 84.
14 H.-X. Mai, L.-D. Sun, Y.-W. Zhang, R. Si, W. Feng, H.-P. Zhang, H.-C. Liu and C.-H. Yan, J. Phys. Chem. B, 2005, 109, 24380-24385.

15 R. Si and M. Flytzani-Stephanopoulos, Angew. Chem., Int. Ed., 2008, 47, 2884-2887.

16 C. Xia, C. Hu, P. Chen, B. Wan, X. He and Y. Tian, Mater. Res. Bull., 2010, 45, 794-798.

17 L. M. Otter, M. W. Förster, E. Belousova, P. O'Reilly, D. Nowak, S. Park, S. Clark, S. F. Foley and D. E. Jacob, Geostand. Geoanal. Res., 2021, 45, 5-27.

18 C. J. Allender, J. L. Bowen, V. Celorrio, J. A. Davies-Jones, P. R. Davies, S. Guan, P. O'Reilly and M. Sankar, Nanoscale Res. Lett., 2020, 15, 161.

19 X. Wen, C. Li and F. Meng, J. Wuhan Univ. Technol., Mater. Sci. Ed., 2014, 29, 229-232.

20 N. Fairley, V. Fernandez, M. Richard-Plouet, C. GuillotDeudon, J. Walton, E. Smith, D. Flahaut, M. Greiner, M. Biesinger, S. Tougaard, D. Morgan and J. Baltrusaitis, Applied Surface Science Advances, 2021, 5, 100112.

21 J. Jahng, D. A. Fishman, S. Park, D. B. Nowak, W. A. Morrison, H. K. Wickramasinghe and E. O. Potma, Acc. Chem. Res., 2015, 48, 2671-2679.

22 D. Nowak, W. Morrison, H. K. Wickramasinghe, J. Jahng, E. Potma, L. Wan, R. Ruiz, T. R. Albrecht, K. Schmidt, J. Frommer, D. P. Sanders and S. Park, Sci. Adv., 2016, 2, e1501571.

23 P. Li, Y. Zhou, Z. Zhao, Q. Xu, X. Wang, M. Xiao and Z. Zou, J. Am. Chem. Soc., 2015, 137, 9547-9550.

24 L. He, Y. Ren, Y. Fu, B. Yue, S. C. E. Tsang and H. He, Molecules, 2019, 24, 526.

25 A. Hezel and S. D. Ross, Spectrochim. Acta, 1966, 22, 1949-1961.

26 F. Bozon-Verduraz and A. Bensalem, J. Chem. Soc., Faraday Trans., 1994, 90, 653-657.

27 Q. Wu, F. Zhang, P. Xiao, H. Tao, X. Wang, Z. Hu and Y. Lü, J. Phys. Chem. C, 2008, 112, 17076-17080.

28 H. X. Mai, L. D. Sun, Y. W. Zhang, R. Si, W. Feng, H. P. Zhang, H. C. Liu and C. H. Yan, J. Phys. Chem. B, 2005, 109, 24380-24385.

29 C. Dong, Y. Zhou, N. Ta and W. Shen, CrystEngComm, 2020, 22, 3033-3041. 